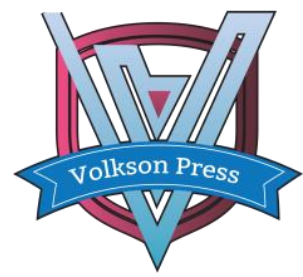

Contents List available at VOLKSON PRESS

Economics \& Management Innovations(EMI)

DOI : http://doi.org/10.26480/icemi.01.2017.370.372

\title{
Automatic Cat Feeding Machine using Internet of Things Technology
}

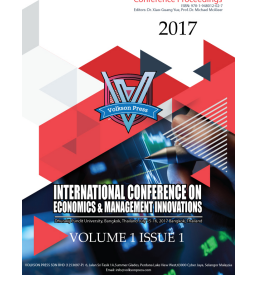

\author{
Wannapa Mokhuntod, Narongdech Keeratipranon and Paethai Akarajariyakul \\ * Department of Computer Engineering and Telecommunications College of Innovative Technology and Engineering Dhurakij Pundit \\ University, Bangkok,Thailand \\ *paethai.akl@dpu.ac.th
}

This is an open access article distributed under the Creative Commons Attribution License, which permits unrestricted use, distribution, and reproduction in any medium, provided the original work is properly cited.

\section{ARTICLE DETAILS}

\section{Article History:}

Received 02 october 2017 Accepted 06 october 2017 Available online 11 october 2017

\section{Keywords:}

Cat Feeding Machine, Amazon Elastic Compute Cloud (EC2), Web Application..

\section{ABSTRACT}

The purpose of this paper is to study and develop a basic automatic cat feeding control system for cat owners that can feed cats in a timely manner. It uses the principle of Real Time Clock module to set the feeding time automatically, Servo Motor to dispense cat food, 2 Motion sensors to monitor frequency and times of the cats approaching the machine and Ultrasonic Distance Measuring Sensors to analyze the amount of remaining food for the next food shopping decision. All generated data from the system are stored on Amazon Elastic Compute Cloud: EC2 and be displayed as graphs, such as the amount of food, the food dispensation status, and the number of times cats approached food through Web Application. The features of this project are that it is low cost and provides cat owners to monitor and manage the feeding system automatically through web and mobile applications.

\section{Introduction}

According to [1], the most common form of malnutrition encountered in small pets is obesity caused by owners' feeding behavior, such as giving excessive amount of food to their pets or untimely feeding due to the forgetfulness. Automatic feeding machines can reduce the problems and also improve cat's behavior [2].

In general, cats are mostly independent and may judge the behavior of their owners based on the manner of time of their feeding. Untimely feeding may result in distrust of their owners as well as mental and physical disorder. Therefore, feeding cats in a timely manner is an essential factor to raise cats successfully and earn their trust, eventually leading to a healthy mutual relationship between cats and their owners. On the other hand, untimely feeding may result in abnormal weight, lack of confidence, and change in behavior. This can be attributed to, but is not limited to, lack of time, social or work obligations of their owners, waking up late, forgetfulness or other issues that may cause the owners to be away.

The purpose of this paper is as follows:

1) To develop automatic cat feeding control system;

2) To design and develop data transmission of time amount of food through Gateway to the Server;

3) To design and develop command system and display of food dispensation status through web and mobile applications;

4) To study the function of Motion Sensor and Ultrasonic Distance MeasuringSensors.

This paper will discuss how technologies are used to ensure that pets are fed properly, sufficiently, and in a timely manner. The second part will discuss relevant studies, while the third part will show how the experiment was carried out. The fourth part will summarize the results of the experiment.

\section{Related Theories}

\subsection{Research Overview}

[3] Automatic Prawn Feeding System was made by Wallop Chandrakul of the Faculty of Technical Education, King Mongkut's University of
Technology North Bangkok. The purpose was to create and evaluate the effectiveness of an automatic feeding system with the scope of creating a prototype controlled with a micro-controller. The system had several features, such as directional control, automatic feeding system, and alert when the food was about to run out. It could also set and control the amount of feed, as well as time and frequency of feeding. The entire system was driven by an electric motor. The results of the experiment with prawns raised within a pond found that the system mostly answered the requirements of the farm entrepreneur.

[4] Automatic Fish Feeding System was created by Jirawuth Sattanako, Piya Yowa, Yutthana Sakkaree and Saruchai Pinakapang of the Electronic Industrial Technology Programme, Faculty of Science, Rajabhat Maha Sarakham University. The purpose was to design and create an automatic fish feeding system that was productive, efficient, and capable of feeding at the set time at the appropriate amount for the age of each fish type. The device can be set to feed the fish up to 5 times per day with 7 segments to display the time and the amount of feeding times. The device was capable of feeding fish thoroughly, resulting in fish of similar size that fetched high prices on the market. The device was also capable of working automatically, even in the event of an electric outage.

[5] The study of design and possibility of automatic dog feeding system was conducted by Pinyo Chanchaisompop of the Business Technology Programme, Faculty of Science, Institute of Field Robotics. The purpose was to study an automatic dog feeding system in order to provide convenience for dog owners. The paper used business and financial analysis to study the return rate of the investment. The survey found out that of 102 dog owners, there were 98 owners who never used an automatic dog feeding system because the device could not be controlled through mobile application. The paper concluded that mobile application control is of wide interest, and the automatic dog feeding system is in high demand and worth investing.

[6] Automatic Dog Feeding System was created by Pratheep Tippracha, Banjerd Ponkarat and Seri Tongchum of the Mechanical Engineering Programme, Faculty of Engineering, Rajamangala University of Technology Srivijaya. The rationale behind the system was the lack of time on dog owner's part, resulting in starving pets that may attack its owner when desperately hungry, resulting in the idea of feeding dogs with an appropriate amount of food at a set time when the dog requested food. Time of feeding can be adjusted according to the owner's needs. By storing dog food in the device's storage tank, and setting the feeding time, feed will be dispensed when the dog comes into contact with the feeding 
tray, which lessens the owner's burden.

\subsection{Arduino}

[7] is a Microcontroller Board of AVR which has Open Source development in both hardware and software. Arduino is suitable for beginners due to its simplicity, and could be easily improved upon, and implemented additional hardware and software.

\subsection{NodeMCU}

[8] is improved upon version .0.9It is a module that consists of ESP128266E with PCB Antenna to connect each individual attenna and connect to the computer for software development. It has $10 \mathrm{GPIO}$ ports, appropriate for IoT usage in order to connect with other devices.

\subsection{Servo Motor}

[9] is used for automatic control. Servo stands for Servus, or a slave in Latin. Servo Motor is a motor that could be programmed and controlled to move in desired direction and angle, and is widely used for radiocontrolled devices.

1) How Servo Motor Works: When pulse signal is transmitted into Servo Motor, the control circuit will read and process the width to rotate the motor to the angle value translated from the pulse signal with sensor to detect the angle.

2) Controlling Servo Motor with Arduino: Arduino controls Servo Motor by rotating from 0 to 180 degree or from 180 to 0 degree. Arduino UNO supports Servo Motor connection up to 12 ports.

3) Functions in Servo Motor'sLibrary: attach() adjusts the width of the antennae and the pulse at 0 degree; write( $)$ adjusts the position according to the set angle; writeMicroseconds 0 adjusts the width of pulse in US unit;

read 0 reads the angle from last set write() function; attached() verifies whether Servo Motor is connected to Arduino; detach() return status from attached() function.

[10] is like a Digital clock that can show temperature. It can be adjusted to show time and date in both 24 hours or 12 hours format, and has internal circuits that can calculate frequency from the environment's temperature that interferes with the Crystal.

\subsection{Motion Sensor}

[11] detects motion from heat. When a living organism moves past, there will be a signal light. Detection range is from 3 to 7 meters. It can also detect at night upon adding a Light Dependent Resistor.

Ultrasonic Module Distance Measuring Transducer Sensor

[12] is used to measure distance. It works by transmitting command signal to trigger and receive signals back for processing at Echo.

\section{MQTT protocol}

[13] Message Queuing Telemetry Transport is a Protocol for MachineTo-Machine Interface. It supports Internet of Things (IoT) technologies that connect objects, such as mobile phone, car, television and refrigerator to the internet, so that the internet network may interface with the object itself remotely.

MQTT Protocol is appropriate for small electronic device in small network with low bandwidth. The functions of the protocol are as follows:

1) Transmit data to a particular Topic (Publisher);

2) Monitor the change in message referenced by Topic in order to use such data (Subscriber);

3) Act as mediator that manage Message by referencing to the Topic (Broker).
[14] is a Cloud system by Amazon. The official name is Amazon Web Services, with a variety of services on the system. This project will use the service of Amazon Elastic Compute Cloud or EC.2 EC2 works by creating Virtual Server that allows the user to select how much resources are needed, such as CPU, Memory and Disk.

\section{Methodology}

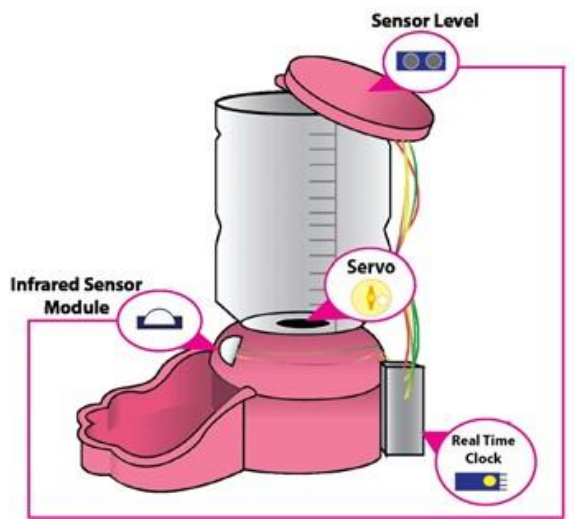

Figure.1 Cat Feeding Machine and Kits

This experiment designed an automatic cat feeding system using 2 sensor nodes to receive data, such as the amount of remaining food and the amount of time cats approached the feeding tray. The data will be transmitted to the gateway, then AWS IoT using rules to store the data on DynamoDB database. The stored data includes the approach movement of the cat, feed dispensation, amount of remaining food in the device, and timestamp. When accessed, the web server will retrieve data from the database, such as the amount of food, cat approach detection, feed dispensation to be displayed on a browser publically, and transmit the data to AWS IoT's Shadows.

Gateway will retrieve data from AWS IoT's Shadows and transmit the data to the Sensor Node in the feeding device to display the status of the device, such as the amount of food remaining, the amount of time the cat approached, and food dispensation.

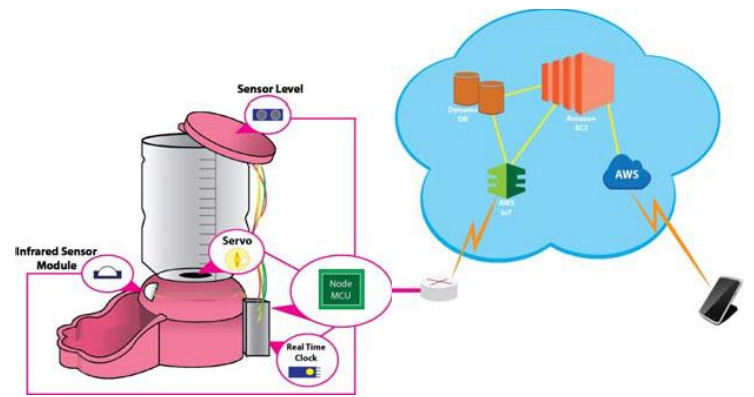

Figure.2 Overall system of Cat Feeding Machine This experiment used Arduino Uno as 2 sets of Sensor Nodes, as follows:

1) Sensor Node to count the amount of food with Ultrasonic sensor;

2) Sensor Node to dispense food according to the set time, consists of the following components: Real Time Clock, Servo Motor, NodeMCU to serve as the Gateway.

Functions:

A. When commanded to be turned on through Web Application, the NodeMCU will transmit turn on command to the Servo Motor and transmit data to AWS IoT;

B. Upon reaching the set time in Real Time Clock, the feeding device will turn on and transmit data to NodeMCU in order to transmit the data further to AWS IoT;

C. Rules on AWS IoT are used for receiving and storing data in the DynamoDB database;

D. Web server will display information on the web application, such as the amount of food dispensation time, the amount of food in the system, and the amount of time a cat approached the device.

\section{Results}


The results of the automatic feeding system are as follows:

4.1 Time setting for automatic feeding: Real Time Clock setting allowed the device to effectively dispense the feed using Servo Motor in a timely manner.

4.2 Feed dispensation: Feed dispensation process of the Servo Motor interacted and worked in accordance with the Real Time Clock, and could be effectively commanded through Web Application.

4.3 Web Application display: Display the remaining amount of food, detection of cat approaching the feeding device and the $d$ spensation status of the Servo Motor. Figure. 3 shows the results of the remaining food.

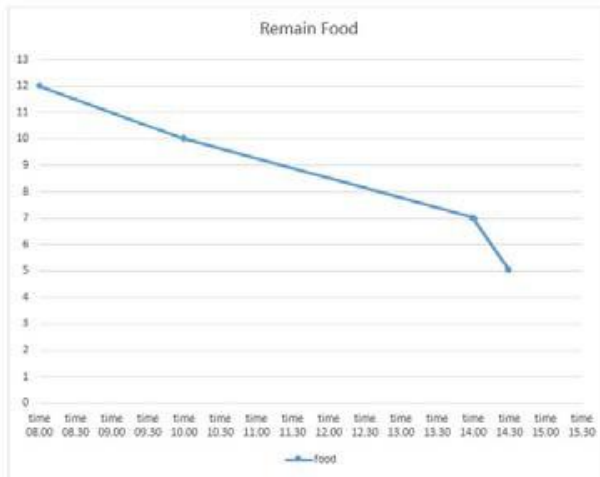

Figure.3 Remains of Food

3) Commanding NodeMCU from the Web Application

4) Uploading information to Cloud through AWS IoT.

\section{Discussion and Conclusion}

From this experiment, the working process of the system proceeded well from the beginning until the end. The system is capable of feeding cats automatically. Nevertheless, the system still experienced error regarding the size of cat feed, and sometimes cat feed stuck, while the servo motor was working. The research team, therefore, recommended that the cat feed used for the system should be of average-sized, not too small or too big.

\section{References}

[1] C. Sloth, "Practical management of obesity in dogs and cats," Journal of Small Animal Practice, vol. 33, pp. 178-182, 1992.

[2] S. Munkevics and M. Munkevics, "Pet-happy," 9 June 2016. [Online]. Available: http://www.pet-happy.com/electronic-cat-feeders-providetwo-huge-behavioral-benefits/. [Accessed 10 March 2017].

[3] W. Chandrakul, "Automatic Prawn Feeding System." Faculty of Technical Education, King Mongkut's University of Technology North Bangkok.

[4] J. Sattanako, P. Yowa, Y. Sakkaree and S. Pinakapang, "Automatic Fish Feeding System." Electronic Industrial Technology Programme, Faculty of Science, Rajabhat Maha Sarakham University.

[5] P. Chanchaisompop, "The study of design and possibility of automatic dog feeding system." Business Technology Programme, Faculty of Science, Institute of Field Robotics.

[6] P. Tippracha, B. Ponkarat and S. Tongchum. "Automatic Dog Feeding System." Faculty of Engineering, Rajamangala University of Technology Srivijaya.

[7] Information on https://www.arduino.cc/. [Accessed 25 May 2017].

[8] Information on http://nodemcu.com/index_en.html. [Accessed 25 May 2017].

[9]

Information

on

http://www.princeton.edu/ mae412/TEXT/NTRAK2002/292-302.pdf [Accessed 18 May 2017].

[10] Information on https://www.engineersgarage.com/real-time-clock [Accessed 25 May 2017]. [11] Information on https://www.mpja.com/download/31227sc.pdf [Accessed 25 May 2017].

Information

on http://www.thaieasyelec.com/products/sensors/infraredultrasonic/us-015-ultrasonic-module-d etail.html [Accessed 25 May 2017].

[13]Information on http://mqtt.org/ [Accessed 25 May 2017].

[14]Information on

http://thai-amazon-cloud.blogspot.com/2014/12/amazon-aws-cloudamazon-web-services.html [Accessed 25 May 2017]. 